\title{
Convergence Rates of Parabolic Difference Schemes for Non-Smooth Data*
}

\author{
By Vidar Thomée and Lars Wahlbin
}

\begin{abstract}
Consider the approximate solution of the initial-value problem for a parabolic system by means of a parabolic finite difference scheme of accuracy $\mu$. The main result of the present paper is essentially that for positive time and $v$ in $W_{1}{ }^{8}$ with $1<s \leq \mu$, the error in the maximum norm is $O\left(h^{8}\right)$ for small mesh-widths $h$.
\end{abstract}

0. Introduction. Consider the initial-value problem for the heat equation

$$
\begin{aligned}
\partial u / \partial t & =\partial^{2} u / \partial x^{2}, \quad x \in R, \quad t>0, \\
u(x, 0) & =v(x),
\end{aligned}
$$

and a consistent single step finite-difference operator with constant coefficients,

$$
E_{k} v(x)=\sum_{i \in Z} a_{j} v(x+j h),
$$

which is accurate of order $\mu$ and parabolic in the sense of John (cf. Section 2).

The problem of estimating, for non-smooth initial data $v$, the rate of convergence of the finite-difference solution $E_{k}{ }^{n} v$ to the solution $E(t) v$ of the continuous problem for $n k=t$ as $k$ and $h$ tend to zero while $\lambda=k h^{-2}$ is kept constant, has been discussed, e.g. in John [5], Juncosa and Young [6], Hedstrom [7], Kreiss, Thomée and Widlund [8], Löfström [9], Peetre and Thomée [10], Wasow [11], Widlund [12], [13]. In particular, it was proved by Löfström [9] that given $1 \leqq p \leqq \infty, 0<s \leqq \mu$, there exists a constant $C$ such that, for $n k>0$,

$$
\left\|E_{k}^{n} v-E(n k) v\right\|_{p} \leqq C h^{s}\|v\|_{p, s}
$$

Here $\|\cdot\|_{p}$ and $\|\cdot\|_{p, s}$ denote the norms in $L_{p}$ and in the Besov space $B_{p}{ }^{s}$, respectively (cf. Section 1). This result was generalized by Widlund [13] to general systems with variable coefficients which are parabolic in Petrovskiı's sense, and correspondingly more general difference operators.

It was also proved in [9] that the estimate $(0.4)$ is best possible in the sense that if $v$ is such that, for $0<n k \leqq T$,

$$
\left\|E_{k}^{n} v-E(n k) v\right\|_{p} \leqq C h^{s},
$$

then $v$ belongs to $B_{p}{ }^{s}$.

In the proof of this latter result, it is essential that the estimate $(0.5)$ holds uniformly for $t=n k$ small. For $p=\infty$, we shall treat the analogous question for $t$ bounded

Received March 8, 1973.

AMS (MOS) subject classifications (1970). Primary 65M10.

* This paper was supported in part by the National Science Foundation and in part by the United States Army under Contract No. DA-31-124-ARO-D-462.

Copyright (C) 1974, American Mathematical Society 
away from zero. It was proved by Hedstrom [7] that the estimate (0.4) is still best possible in the sense that there exists a function in $B_{\infty}{ }^{8}$ for which the error is bounded below by a positive multiple of $h^{*}$ for a sequence of arbitrarily small $h$. For an individual $v$, however, we shall now only be able to prove that the estimate (0.5) implies that $v$ belongs to $B_{\infty}{ }^{s-1}$. We shall further show the positive result that, given $1<s \leqq \mu$, there exists a constant $C$ such that, for $n k>0$,

$$
\left\|E_{k}^{n} v-E(n k) v\right\|_{\infty} \leqq C h^{s}(n k)^{-1 / 2}\|v\|_{1, s} .
$$

This result is of interest for instance when data have singularities only at a finite number of points. Such a function in $B_{\infty}{ }^{s}$ may then belong to $B_{1}{ }^{s+1}$, but not to $B_{\infty}{ }^{8+c}$ for any $\epsilon>0$ (cf. Example 1.1) so that the estimate (0.6) then shows an additional power of $h$ convergence rate in the maximum norm for $t$ bounded away from zero. In a special case, a similar result was proved by Juncosa and Young [6].

Following some preliminaries in Section 1, these results will be proved by Fourier methods in Section 2. In Section 3, the estimate $(0.6)$ is generalized to the more general context treated by Widlund in [12] and [13]. The proof depends heavily on these papers.

In Kreiss, Thomée and Widlund [8], it was noticed that an appropriate smoothing of nonsmooth initial data can improve the convergence rate for $t$ bounded away from zero. In Section 4, we combine the ideas of that paper with those of Section 3.

Throughout this paper, $C$ and $c$ denote large and small positive constants, respectively, not necessarily the same at different occurrences.

1. Preliminaries. We define the Fourier transform and its inverse on $L_{1}\left(R^{d}\right)$ by

$$
\begin{aligned}
\mathfrak{F} v(\xi) & =\hat{v}(\xi)=\int \exp (-i\langle x, \xi\rangle) v(x) d x, \quad\langle x, \xi\rangle=\sum_{i=1}^{d} x_{i} \xi_{i}, \\
\mathcal{F}^{-1} v(x) & =\breve{v}(x)=(2 \pi)^{-d} \int \exp (i\langle x, \xi\rangle) v(\xi) d \xi .
\end{aligned}
$$

This definition extends to tempered distributions, in particular, to $L_{p}, 1 \leqq p \leqq \infty$ (cf. [4, Chapter 1]).

We shall define the Besov spaces $B_{p}{ }^{s}$ via a partition of unity on the dual $R^{d}$. An equivalent definition in terms of moduli of continuity in $L_{p}$ can be found in [9] (where $B_{p}{ }^{*}$ is denoted $B_{p}{ }^{s, \infty}$ ) and a proof of the equivalence in [3].

Let $\varphi$ be a nonnegative $C^{\infty}$ function with support in $\left\{\xi ; \frac{1}{2}<|\xi|<2\right\}$ such that

$$
\sum_{i \in Z} \varphi\left(2^{-i} \xi\right)=1 \text { for } \xi \neq 0 .
$$

We set

$$
\begin{aligned}
& \varphi_{i}(\xi)=\varphi\left(2^{-i} \xi\right), \quad j=1,2, \cdots, \\
& \varphi_{0}(\xi)=1-\sum_{j=1}^{\infty} \varphi_{i}(\xi) .
\end{aligned}
$$

Let $1 \leqq p \leqq \infty$ and $s>0$ be given. The space $B_{p}{ }^{*}$ is the space of functions $v$ in $L_{p}$ such that with $v_{j}=\check{\varphi}_{i} * v=\mathfrak{F}^{-1}\left(\varphi_{i} \hat{v}\right)$ (this notation will be used throughout this paper) we have

$$
\|v\|_{p, s}=\sup _{i \geqq n} 2^{s i}\left\|v_{j}\right\|_{p}<\infty
$$


The parameter $s$ measures the smoothness of the elements in $B_{p}{ }^{8}$; the space $B_{p}{ }^{*}$ decreases as $s$ increases and for $s$ integer and any $\epsilon>0$, we have $B_{p}{ }^{\circ} \supseteq W_{p}{ }^{\circ} \supseteq B_{p}{ }^{*+\epsilon}$ where $W_{p}{ }^{3}$ is the Sobolev space of functions with derivatives of orders up to and including $s$ in $L_{p}$. We notice that by the Sobolev embedding theorem, if $v$ belongs to $B_{1}{ }^{s}$ for some $s>d$, then $v$ belongs to the set $\mathfrak{C}_{0}$ of continuous functions which vanish at infinity and there is a constant $C$ such that $\|v\|_{\infty} \leqq C\|v\|_{1, s}$.

For later reference, we present two one-dimensional examples.

Example 1.1. Let $\chi \in C_{0}{ }^{\infty}$ and $\chi(0) \neq 0$, and set, for $\sigma>0$,

$$
\begin{aligned}
\chi_{\sigma}(x) & =x^{\sigma} \chi(x), & & x \geqq 0, \\
& =0, & & x<0 .
\end{aligned}
$$

Then $\chi_{\sigma} \in B_{p}{ }^{s}$ if and only if $s \leqq \sigma+p^{-1}$ as is easily seen using the equivalent norm in $B_{p}{ }^{3}$ in terms of moduli of continuity. Many functions in applications are linear combinations of translates of functions of this type.

Example 1.2. Let $\psi$ be such that $\hat{\psi} \in C_{0}{ }^{\infty}(0,1)$, and set, for $\sigma>0$,

$$
\psi_{\sigma}(x)=\left(\sum_{i=0}^{\infty} \exp \left(i x 2^{j}\right) 2^{-\sigma i}\right) \psi(x),
$$

so that

$$
\hat{\psi}_{\sigma}(\xi)=\sum_{j=0}^{\infty} 2^{-\sigma j} \hat{\psi}\left(\xi-2^{i}\right)
$$

Notice that the supports of the different terms in $\hat{\psi}_{\sigma}$ are disjoint. It is easy to see that, for any $p, \psi_{\sigma} \in B_{p}^{s}$ if and only if $s \leqq \sigma$. This function is not likely to occur in applications. For $\sigma$ a nonzero integer, $D^{\sigma-1} \psi_{\sigma}$ is continuous but $D^{\sigma} \psi_{\sigma}$ is nonexistent a.e. (cf. [1, p. 265]).

We conclude this section with two elementary lemmas.

LEMMA 1.1. There exists a constant $C$ such that, for any $a \in \mathfrak{C}_{0}^{\infty}$,

$$
\left\|\mathcal{F}^{-1}(a \hat{v})\right\|_{p} \leqq C M(a)\|v\|_{p}, \quad p=1, \infty,
$$

where $M(a)=\left(\|a\|_{2} \max _{|\alpha|=d}\left\|D^{\alpha} a\right\|_{2}\right)^{1 / 2}$.

Proof. For $a \in \mathcal{C}_{0}{ }^{\infty}$, we have clearly $\check{a} \in L_{1}$ and, for $p=1, \infty$,

$$
\left\|F^{-1}(a \hat{v})\right\|_{p}=\|\check{a} * v\|_{p} \leqq\|\check{a}\|_{1}\|v\|_{p} .
$$

Using Hölder's inequality and Parseval's relation, we obtain

$$
\begin{aligned}
\|\check{a}\|_{1} & =\int_{|x| \leqq \delta}|\check{a}(x)| d x+\int_{|x|>\delta}|\check{a}(x)||x|^{d}|x|^{-d} d x \\
& \leqq C\left\{\delta^{d / 2}\|a\|_{2}+\delta^{-d / 2} \max _{|\alpha|=d}\left\|D^{\alpha} a\right\|_{2}\right\} .
\end{aligned}
$$

The lemma now follows by choosing, for $a \neq 0$,

$$
\delta=\|a\|_{2}^{-1 / d}\left(\max _{|\alpha|=d}\left\|D^{\alpha} a\right\|_{2}\right)^{1 / d} .
$$

Lemma 1.2. For $a \in \mathcal{C}_{0}^{\infty}$, we have 


$$
\left\|\mathcal{F}^{-1}(a \hat{v})\right\|_{\infty} \leqq(2 \pi)^{-d}\|a\|_{1}\|v\|_{1} .
$$

Proof. We have here

$$
\|\check{a} * v\|_{\infty} \leqq\|\check{a}\|_{\infty}\|v\|_{1} \leqq(2 \pi)^{-d}\|a\|_{1}\|v\|_{1} .
$$

2. The One-Dimensional Heat Equation. Consider the initial-value problem $(0.1),(0.2)$ with solution

$$
\begin{aligned}
u(x, t) & =E(t) v(x)=\frac{1}{(4 \pi t)^{1 / 2}} \int \exp \left(-\frac{(x-y)^{2}}{4 t}\right) v(y) d y \\
& =F^{-1}\left(\exp \left(-t \xi^{2}\right) \hat{v}\right)(x)
\end{aligned}
$$

Consider also the consistent finite-difference operator (0.3). Introducing its characteristic function, which we assume to be analytic,

$$
a(\omega)=\sum_{i \in Z} a_{i} \exp (i j \omega),
$$

the discrete solution operator at $t=n k$ can be represented as

$$
E_{k}^{n} v=\mathcal{F}^{-1}\left(a(h \xi)^{n} \hat{v}\right)
$$

We shall assume that $E_{k}$ is accurate of order $\mu$ so that

$$
a(\omega)-\exp \left(-\lambda \omega^{2}\right)=r(\omega)=O\left(|\omega|^{2+\mu}\right) \text { as } \omega \rightarrow 0 .
$$

We shall also assume that $E_{k}$ is parabolic in the sense of John [5], i.e., that there exists a constant $c>0$ such that

$$
|a(\omega)| \leqq \exp \left(-c \omega^{2}\right) \quad \text { for }|\omega| \leqq \pi .
$$

For the sake of completeness, we shall first give a proof of the sharpness of the $O\left(h^{3}\right)$ error estimate (0.4) for $t \neq 0$ when $v$ is allowed to vary over $B_{\infty}{ }^{*}$ (cf. Hedstrom [7, Theorem 10.2]).

THEOREM 2.1. Let $0<s \leqq \mu$, and $t>0$. Then there exists a function $v \in B_{\infty}{ }^{s}$ such that

$$
\limsup _{h \rightarrow 0 ; n k=t} h^{-s}\left\|E_{k}^{n} v-E(t) v\right\|_{\infty}>0 .
$$

Proof. Without loss of generality, we take $t=1$. We choose $v$ to be the function $\psi_{\text {a }}$ of Example 1.2, and set

$$
\epsilon_{p, h}=\left\|\left(E_{k}^{n}-E(1)\right) \psi_{s}\right\|_{p}, \quad n k=1 .
$$

By Hölder's inequality and application of $(0.4)$ with $p=1$, we obtain

$$
\epsilon_{2, h}^{2} \leqq \epsilon_{1, h} \epsilon_{\infty, h} \leqq C h^{\circ} \epsilon_{\infty, h} .
$$

The conclusion of the theorem hence follows if we can prove that

$$
\limsup _{h \rightarrow 0} h^{-s} \epsilon_{2, h}>0 .
$$

Using Parseval's relation, the definition of $\psi_{\mathrm{s}}$ and the periodicity of $a$, we obtain for any nonnegative integer $m$, with $I_{m}=\left(2^{m}, 2^{m}+1\right)$, 


$$
\begin{aligned}
\epsilon_{2, h}^{2} & =(2 \pi)^{-1} \int_{-\infty}^{\infty}\left|a(h \xi)^{n}-\exp \left(-\xi^{2}\right)\right|^{2}\left|\hat{\psi}_{s}(\xi)\right|^{2} d \xi \\
& \geqq(2 \pi)^{-1} \int_{I_{m}}\left|a(h \xi-2 \pi)^{n}-\exp \left(-\xi^{2}\right)\right|^{2} 2^{-2 s m}\left|\hat{\psi}\left(\xi-2^{m}\right)\right|^{2} d \xi .
\end{aligned}
$$

We now choose $h_{m}=\left(\lambda n_{m}\right)^{-1 / 2}$ where $n_{m}$ is the integral part of $(2 \pi)^{-2} \lambda^{-1} 2^{2 m}$ so that $\left|2 \pi h_{m}{ }^{-1}-2^{m}\right| \leqq 2 \pi \lambda^{1 / 2}$. For $\xi$ in $I_{m}$ it follows that $\left|h_{m} \xi-2 \pi\right| \leqq\left(1+2 \pi \lambda^{1 / 2}\right) h_{m}$ and hence for large $m$, by consistency,

$$
\inf _{I_{m}}\left|a\left(h_{m} \xi-2 \pi\right)^{n_{m}}\right| \geqq \exp \left(-c n_{m} h_{m}^{2}\right) \geqq c>0 .
$$

Since also $\lim _{m \rightarrow \infty} \sup _{I_{m}} \exp \left(-\xi^{2}\right)=0$, we obtain from (2.1), for large $m$,

which concludes the proof.

$$
\epsilon_{2, h_{m}}^{2} \geqq c 2^{-2 s m} \geqq c h_{m}^{2 s},
$$

We shall now show that for an individual $v$, an $O\left(h^{s}\right)$ maximum norm convergence rate estimate for a fixed positive $t$ implies that $v \in B_{\infty}{ }^{8-1}$.

THEOREM 2.2. Let $1<s \leqq \mu$, and $t>0$. Assume that for a fixed function $v$ in $L_{\infty}$ there is a constant $C$ such that, for $n k=t$,

$$
\left\|E_{k}^{n} v-E(t) v\right\|_{\infty} \leqq C h^{\circ} .
$$

Then $v \in B_{\infty}^{8-1}$.

Proof. Without loss of generality, we take $t=1$. Let $f$ be a nonnegative $C_{0}{ }^{\infty}$ function with support in $(-1,1)$ such that with $f_{m}(\xi)=f(\xi-m)$ for $m$ integer,

For $j>0$, we may then write

$$
\sum_{m=-\infty}^{\infty} f_{m}(\xi)=1
$$

$$
\left\|v_{i}\right\|_{\infty} \leqq \sum_{2^{i-1} \leqq|m| \leqq 2^{i+1}}\left\|F^{-1}\left(\varphi_{i} f_{m} \hat{v}\right)\right\|_{\infty} .
$$

For the purpose of estimating the terms in (2.3), consider now $j$ and $m$ satisfying

$$
2^{i-1} \leqq m \leqq 2^{i+1} \text {. }
$$

(Negative $m$ can be treated in the same way.) Let $j_{1} \geqq 1$ be such that for $j \geqq j_{1}$ there exists, for any $m$ satisfying (2.4), an integer $n_{m}$ with

$$
(m-1)^{2} /\left(\lambda(2 \pi)^{2}\right)<n_{m}<(m+1)^{2} /\left(\lambda(2 \pi)^{2}\right) .
$$

Setting $h_{m}=\left(\lambda n_{m}\right)^{-1 / 2}$, we have, for $\xi$ in supp $f_{m}$,

$$
-2<\xi-2 \pi h_{m}^{-1}<2 \text {. }
$$

Let $e_{m}(\xi)=a^{n_{m}}\left(h_{m} \xi\right)-\exp \left(-\xi^{2}\right)$. We shall show that for $j$ large enough $e_{m}{ }^{-1}$ exists on supp $f_{m}$ and that, with the notation of Lemma 1.1,

$$
M\left(f_{m} e_{m}^{-1}\right) \leqq C .
$$

By periodicity and (2.5), we have, in supp $f_{m}$ (cf. (2.2)),

$$
\left|a^{n_{m}}\left(h_{m} \xi\right)\right|=\left|a^{n_{m}}\left(h_{m} \xi-2 \pi\right)\right| \geqq \exp \left(-c \lambda^{-1}\right)>0 .
$$

Since $\exp \left(-\xi^{2}\right)$ is small in supp $f_{m}$ if $j$ is large, we see that there exists $j_{2} \geqq j_{1}$ such that for $j \geqq j_{2}, e_{m}{ }^{-1}$ exists on $\operatorname{supp} f_{m}$ and 


$$
\left\|f_{m} e_{m}^{-1}\right\|_{2} \leqq C \text {. }
$$

Since, by consistency, $\left|a^{\prime}(\omega)\right|=O(|\omega|)$ as $\omega$ tends to 0 , we obtain, using the periodicity and (2.5),

$$
\left|D e_{m}^{-1}(\xi)\right|=\left|n_{m} a^{n_{m}-1}\left(h_{m} \xi\right) h_{m} a^{\prime}\left(h_{m} \xi\right)+2 \xi \exp \left(-\xi^{2}\right)\right|\left|e_{m}^{-2}\right| \leqq C .
$$

Hence

$$
\left\|D\left(f_{m} e_{m}^{-1}\right)\right\|_{2} \leqq C
$$

Together, (2.7) and (2.8) prove (2.6).

We now note that, for $j \geqq 2, h_{m} \leqq 2 \pi\left(2^{i-1}-1\right)^{-1} \leqq 8 \pi 2^{-i}$, and that by a simple application of the definition, for $j>0, M\left(\varphi_{j}\right)=M(\varphi)=C$. Hence, by Lemma 1.1, (2.6) and by the hypothesis of the theorem, for $j \geqq j_{0}=\max \left(j_{2}, 2\right)$ with $k_{m}=\lambda h_{m}{ }^{2}$,

$$
\left\|\mathcal{F}^{-1}\left(\varphi_{j} f_{m} \hat{v}\right)\right\|_{\infty} \leqq M\left(\varphi_{i}\right) M\left(f_{m} e_{m}^{-1}\right)\left\|\left(E_{k_{m}}^{n_{m}}-E(1)\right) v\right\|_{\infty} \leqq C h_{m}^{s} \leqq C 2^{-i s} .
$$

Since the summation in (2.3) involves less than $2 \cdot 2^{i+1}$ terms, we obtain

$$
\sup _{j \geqq i_{0}} 2^{i(s-1)}\left\|v_{i}\right\|_{\infty} \leqq C .
$$

Further we have, since $v \in L_{\infty}$,

$$
\max _{i<i_{0}} 2^{i(s-1)}\left\|v_{j}\right\|_{\infty} \leqq 2^{\left(i_{0}-1\right)(s-1)} \max \left(M\left(\varphi_{0}\right), M(\varphi)\right)\|v\|_{\infty} \leqq C .
$$

Together, (2.9) and (2.10) prove the theorem.

We now present the main result of this section which estimates the error in the maximum norm in terms of the smoothness of the initial data measured in $L_{1}$.

THEOREM 2.3. Let $1<s \leqq \mu$. Then there exists a constant $C$ such that, for $v \in B_{1}$,

$$
\left\|E_{k}^{n} v-E(n k) v\right\|_{\infty} \leqq C h^{s}(n k)^{-1 / 2}\|v\|_{1, s}, \quad n=1,2,3, \cdots .
$$

Proof. Defining $\varphi_{-1}=0$, we have in $\operatorname{supp} \varphi_{i}$, for $j \geqq 0$,

$$
\sum_{l=i-1}^{i+1} \varphi_{l}=1
$$

Hence we may write the error in the form

$$
E_{k}^{n} v-E(n k) v=\mathcal{F}^{-1}\left(\sum_{i=0}^{\infty} \varphi_{j} \hat{F}_{h}(n k) \mathcal{F}\left(\sum_{l=i-1}^{i+1} v_{l}\right)\right)
$$

where $\hat{F}_{h}(n k)(\xi)=a(h \xi)^{n}-\exp \left(-n k \xi^{2}\right)$. Using Lemma 1.2, we obtain

$$
\begin{aligned}
\left\|E_{k}^{n} v-E(n k) v\right\|_{\infty} & \leqq \sum_{i=0}^{\infty} \sum_{l=i=1}^{i+1}\left\|\mathcal{F}^{-1}\left(\varphi_{i} \hat{F}_{h}(n k) \hat{v}_{l}\right)\right\|_{\infty} \\
& \leqq(2 \pi)^{-1} \sum_{i=0}^{\infty} \sum_{l=i-1}^{i+1}\left\|\varphi_{i} \hat{F}_{h}(n k)\right\|_{1}\left\|v_{l}\right\|_{1} \\
& \leqq 3 \cdot 2^{s}(2 \pi)^{-1}\left(\sum_{i=0}^{\infty}\left\|\varphi_{i} \hat{F}_{h}(n k)\right\|_{1} 2^{-s i}\right)\|v\|_{1, s}
\end{aligned}
$$

For the purpose of estimating the terms in the latter sum, we shall prove that 


$$
\begin{aligned}
\left\|\varphi_{i} \hat{F}_{h}(n k)\right\|_{1} & \leqq C h^{i}(n k)^{-(s+1) / 2} \min \left(1,\left[2^{i}(n k)^{1 / 2}\right]^{3+s}\right), & & 2^{i} h \leqq \pi / 2, \\
& \leqq C h 2^{i}(n k)^{-1 / 2}, & & 2^{i} h \geqq \pi / 2 .
\end{aligned}
$$

Assuming that these estimates have been proved, we obtain, since $1<s$,

$$
\begin{aligned}
& \sum_{i=0}^{\infty}\left\|\varphi_{i} \hat{F}_{h}(n k)\right\|_{1} 2^{-s i} \\
& \leqq C h^{i}(n k)^{-1 / 2} \sum_{2^{i}(n k)^{1 / 2} \leqq 1}\left(2^{i}(n k)^{1 / 2}\right)^{3} \\
&+C h^{*}(n k)^{-(s+1) / 2} \sum_{2^{i}(n k)^{1 / 2} \geqq 1 ; 2^{i} \leqq \pi / 2 h} 2^{-s i}+C h(n k)^{-1 / 2} \sum_{2^{i} \geqq \pi / 2 h} 2^{i(1-s)} \\
& \leqq C h^{i}(n k)^{-1 / 2},
\end{aligned}
$$

with a constant depending on $s$. By $(2.11)$ this proves the theorem.

It remains to prove the estimates (2.12). Consider first the case $h 2^{i} \leqq \pi / 2$. Using our accuracy and parabolicity assumptions, we obtain for $|h \xi| \leqq \pi$, since $s \leqq \mu$,

$$
\begin{aligned}
\left|\hat{F}_{h}(n k)(\xi)\right| & =\left|\sum_{m=0}^{n-1} a(h \xi)^{n-m-1} r(h \xi) \exp \left(-m \lambda(h \xi)^{2}\right)\right| \\
& \leqq \sum_{m=0}^{n-1} \exp \left(-c(n-m-1)(h \xi)^{2}\right) C|h \xi|^{2+\bullet} \exp \left(-m \lambda(h \xi)^{2}\right) \\
& \leqq C n|h \xi|^{2+s} \exp \left(-c n(h \xi)^{2}\right)=C h^{2} n k|\xi|^{2+\infty} \exp \left(-c n k \xi^{2}\right) .
\end{aligned}
$$

Hence

$$
\begin{aligned}
\left\|\varphi_{i} \hat{F}_{h}(n k)\right\|_{1} & \leqq C h^{\circ} n k \int_{|\xi| \leq 2^{i+1}}|\xi|^{2+\theta} \exp \left(-c n k \xi^{2}\right) d \xi \\
& =C h^{\circ}(n k)^{-(s+1) / 2} \int_{|z| \leq(n k)^{1 / 2} 2^{i+2}}|z|^{2+s} \exp \left(-c z^{2}\right) d z \\
& \leqq C h^{0}(n k)^{-(s+1) / 2} \min \left(1,\left(2^{i}(n k)^{1 / 2}\right)^{3+s}\right) .
\end{aligned}
$$

This proves (2.12) in the case $2^{i} h \leqq \pi / 2$.

Consider now the case $h 2^{i} \geqq \pi / 2$. Obviously, we have

$$
\left\|\varphi_{i} \exp \left(-n k \xi^{2}\right)\right\|_{1} \leqq C(n k)^{-1 / 2} \leqq C(n k)^{-1 / 2} h 2^{i} .
$$

In order to estimate $\varphi_{i} a(h \xi)^{n}$, let

$$
S_{i}=\left\{m \in Z ; h 2^{i-1} \leqq 2 \pi m \leqq h 2^{i+1}\right\} .
$$

We then have, using periodicity and parabolicity,

$$
\begin{aligned}
\left\|\varphi_{i} a(h \xi)^{n}\right\|_{1} & \leqq \sum_{m \in S_{i}} \int_{|n \xi-2 \pi m| \leq \pi}\left|\varphi_{i}(\xi) a(h \xi-2 \pi m)^{n}\right| d \xi \\
& \leqq \sum_{m \in S_{i}} \int_{|\xi| \leqq \pi / h} \exp \left(-c n k \xi^{2}\right) d \xi \\
& \leqq C(n k)^{-1 / 2} \sum_{m \in S_{i}} 1 \leqq C(n k)^{-1 / 2} h 2^{j} .
\end{aligned}
$$


Together, (2.13) and (2.14) prove the desired inequality by use of the triangle inequality.

3. The General Parabolic Case. In this section, the object is to generalize the main result of Section 2, Theorem 2.3, to the case of general parabolic systems and difference operators. For simplicity, we consider only systems with time independent coefficients.

Consider thus the initial-value problem

$$
\begin{gathered}
\frac{\partial u}{\partial t}=P(x, D) u=\sum_{|\alpha| \leq 2 M} P_{\alpha}(x) D^{\alpha} u, \quad x \in R^{d}, \quad t>0, \\
u(x, 0)=v(x),
\end{gathered}
$$

where $u, v$ are $N$-vectors and $P_{\alpha}$ are $N \times N$ matrices which have bounded derivatives of all orders. We assume that (3.1) is parabolic in Petrovskir's sense so that the eigenvalues $\lambda_{j}(x, \xi)$ of $(-1)^{M} \sum_{|\alpha|=2 M} P_{\alpha}(x) \xi^{\alpha}$ satisfy, with $c>0$,

$$
\operatorname{Re} \lambda_{i}(x, \xi) \leqq-c|\xi|^{2 M} \text { for } x, \xi \in R^{d} .
$$

Consider also a difference operator $E_{k}$ approximating the solution operator $E(k)$ of (3.1), (3.2), defined by a difference equation of the form

$$
\sum_{\beta \in Z^{d}} a_{\beta}(x, h)\left(E_{k} v\right)(x+\beta h)=\sum_{\beta \in Z^{d}} a_{\beta}^{\prime}(x, h) v(x+\beta h),
$$

where $k h^{-2 M}=\lambda=$ constant and $a_{\beta}, a_{\beta}{ }^{\prime}$ are $N \times N$ matrices which have bounded derivatives of all orders in $R^{d} \times\left[0, h_{0}\right]$ for some $h_{0}>0$, and of which only finitely many are nonzero. The operator $E_{k}$ will be assumed to be accurate of order $\mu$ so that, for smooth solutions $u$ of (3.1),

$$
u(x, t+k)=E_{k} u(x, t)+k O\left(h^{\mu}\right) \text { as } h \text { tends to } 0 .
$$

We also assume that $E_{k}$ is parabolic in the sense of John so that the eigenvalues $\mu_{i}(x, \omega)$ of the matrix

$$
\left(\sum_{\beta} a_{\beta}(x, 0) \exp (i\langle\beta, \omega\rangle)\right)^{-1}\left(\sum_{\beta} a_{\beta}^{\prime}(x, 0) \exp (i\langle\beta, \omega\rangle)\right)
$$

satisfy, with $c>0$,

$$
\left|\mu_{i}(x, \omega)\right| \leqq \exp \left(-c|\omega|^{2 M}\right) \text { for } \max _{i}\left|\omega_{i}\right| \leqq \pi .
$$

In the technical work, we shall need the following norm:

$$
\|v\|_{(h)}=\sup _{x \in R^{d}} h^{d} \sum_{j \in Z^{d}}|v(x+j h)| .
$$

For the transition between this norm based on $l_{1}$ mesh norms over translated meshes, and $L_{1}$-norms, we have the following estimate.

LEMMA 3.1. There exists a constant $C$ independent of $h$ such that, for all $v \in W_{1}^{d}\left(R^{d}\right)$

$$
\|v\|_{(h)} \leqq C \sum_{|\alpha| \leqq d} h^{|\alpha|}\left\|D^{\alpha} v\right\|_{1}
$$


Proof. We may assume that $v$ is smooth. By homogeneity, it suffices to consider $h=1$. Let $Q$ denote a cube of side 1 , centered at the origin. We then have

$$
|v(0)| \leqq C \sum_{|\alpha| \leqq d} \int_{0}\left|D^{\alpha} v(y)\right| d y .
$$

Hence, for any $x \in R^{d}, j \in Z^{d}$,

$$
|v(x+j)| \leqq C \sum_{|\alpha| \leqq d} \int_{x+i+0}\left|D^{\alpha} v(y)\right| d y,
$$

and the result follows by summation over $j$ and taking supremum in $x$.

The next two lemmas contain estimates for the continuous and discrete solution operators which are simple consequences of known estimates for the fundamental solutions in the two cases.

LEMMA 3.2. Let $0<T_{0}<T_{1}$. Then for any $\alpha$ there exists a constant $C$ such that, for $v \in \mathfrak{C}_{0}^{\infty}$,

$$
\left\|E(t) D^{\alpha} v\right\|_{\infty} \leqq C\|v\|_{1}, \quad T_{0} \leqq t \leqq T_{1} .
$$

Proof. Under our assumptions the initial-value problem (3.1), (3.2) has a fundamental solution $\Gamma(x, t, y)$ such that (cf. [2, pp. 260-261]),

$$
\left|D_{\nu}^{\alpha} \Gamma(x, t, y)\right| \leqq C t^{-(d+|\alpha|) /(2 M)} \exp \left(-c\left(\frac{|x-y|^{2 M}}{t}\right)^{1 /(2 M-1)}\right) .
$$

Now

$$
E(t) D^{\alpha} v(x)=\int \Gamma(x, t, y) D^{\alpha} v(y) d y=(-1)^{|\alpha|} \int D_{\nu}^{\alpha} \Gamma(x, t, y) v(y) d y,
$$

and the result follows immediately.

In the following discrete analogue of Lemma 3.2, we use the notation

$$
\partial_{h}^{\alpha}=\partial_{h, 1}^{\alpha d} \cdots \partial_{h, d}^{\alpha d} \quad \text { with } \partial_{h, j} v(x)=\left(v\left(x+h e_{i}\right)-v(x)\right) / h .
$$

Lemma 3.3. Let $0<T_{0}<T_{1}$. Then, for any $\alpha$, there exists a constant $C$ such that, for $v \in \mathbf{e}_{0}^{\infty}$,

$$
\left\|E_{k}^{n} \partial_{h}^{\alpha} v\right\|_{\infty} \leqq C\|v\|_{(h)}, \quad T_{0} \leqq n k \leqq T_{1} .
$$

Proof. Here, there exists a discrete fundamental solution $\Gamma_{h}(x, n k, y)$ such that (cf. [12])

$$
\left|\partial_{h, \nu}^{\alpha} \Gamma_{h}(x, n k, y)\right| \leqq C((n+1) k)^{(d+|\alpha|) /(2 M)},
$$

so that, by summation by parts,

$$
\begin{aligned}
E_{k}^{n} \partial_{h}^{\alpha} v(x) & =h^{d} \sum_{y \in x+h Z^{d}} \Gamma_{h}(x, n k, y) \partial_{h}^{\alpha} v(y) \\
& =(-1)^{|\alpha|} h^{d} \sum_{y \in x+h Z^{d}} \partial_{h, y}^{\alpha} \Gamma_{h}(x, n k, y) v(y),
\end{aligned}
$$

and again the result follows immediately.

As a final preparation for the main result, we quote the following crucial estimate [13, Theorem 3]. 
LEMMA 3.4. Let $0<T_{0}<T_{1}$. Then, there exists a constant $C$ such that if $v \in \mathfrak{C}_{0}{ }^{-}$ and $\hat{v}(\xi)=0$ for $\max _{i}\left|h \xi_{i}\right| \geqq \pi$, then

$$
\left\|E_{k}^{n} v-E(n k) v\right\|_{\infty} \leqq C h^{\mu}\|v\|_{\infty}, \quad T_{0} \leqq n k \leqq T_{1} .
$$

We now state and prove the generalization of Theorem 2.3.

THEOREM 3.1. Let $d<s \leqq \mu$, and $0<T_{0}<T_{1}$. Then, there exists a constant $C$ such that, for $v \in B_{1}{ }^{3}$,

$$
\left\|E_{k}^{n} v-E(n k) v\right\|_{\infty} \leqq C h^{s}\|v\|_{1, s}, \quad T_{0} \leqq n k \leqq T_{1} .
$$

Proof. We find easily, for $|\alpha| \leqq d$,

$$
\begin{aligned}
\left\|D^{\alpha} v_{i}\right\|_{1} & =\left\|F^{-1} \xi^{\alpha} \sum_{l=i-1}^{i+1} \varphi_{l} \varphi_{j} \hat{v}\right\|_{1} \leqq C \sum_{l=i-1}^{j+1} M\left(\xi^{\alpha} \varphi_{l}\right)\left\|v_{i}\right\|_{1} \\
& \leqq C 2^{i|\alpha|}\left\|v_{i}\right\|_{1} .
\end{aligned}
$$

Put $F_{h}(n k) v=E_{k}{ }^{n} v-E(n k) v$.

For $j$ with $h 2^{i} \leqq \pi / 2$, we have $\hat{v}_{i}=0$ when $\max _{l}\left|h \xi_{l}\right| \geqq \pi$, so that for such $j$ we have, by Lemma 3.4 and (3.3),

$$
\begin{aligned}
\left\|F_{h}(n k) v_{i}\right\|_{\infty} & \leqq C h^{\mu}\left\|v_{i}\right\|_{\infty} \leqq C h^{\mu}\left\|v_{i}\right\|_{W_{i}^{d}} \leqq C h^{\mu} 2^{j d}\left\|v_{i}\right\|_{1} \\
& \leqq C h^{s} 2^{-i(s-d)}\|v\|_{1, s}
\end{aligned}
$$

On the other hand, when $h 2^{i} \geqq \pi / 2$, we have, by Lemma 3.3 (with $\alpha=0$ ), Lemma 3.1 and (3.3),

$$
\begin{aligned}
\left\|E_{k}^{n} v_{j}\right\|_{\infty} & \leqq C\left\|v_{i}\right\|_{(h)} \leqq C \sum_{|\alpha| \leqq d} h^{|\alpha|}\left\|D^{\alpha} v_{i}\right\|_{1} \\
& \leqq C \sum_{|\alpha| \leqq d}\left(h 2^{j}\right)^{|\alpha|}\left\|v_{i}\right\|_{1} \leqq C\left(h 2^{j}\right)^{d}\left\|v_{i}\right\|_{1} \\
& \leqq C h^{d} 2^{-j(s-d)}\|v\|_{1, s} .
\end{aligned}
$$

Also, for these $j$, we have, by Lemma 3.2,

$$
\left\|E(n k) v_{i}\right\|_{\infty} \leqq C\left\|v_{j}\right\|_{1} \leqq C 2^{-i s}\|v\|_{1, s} .
$$

Altogether, we obtain, by (3.4), (3.5) and (3.6),

$$
\begin{aligned}
\left\|F_{h}(n k) v\right\|_{\infty} & \leqq \sum_{i=0}^{\infty}\left\|F_{h}(n k) v_{j}\right\|_{\infty} \\
& \leqq C\left\{h^{s} \sum_{h 2^{i} \leqq \pi / 2} 2^{-i(s-d)}+h^{d} \sum_{h 2^{i}>\pi / 2} 2^{-i(s-d)}+\sum_{h 2^{i}>\pi / 2} 2^{-i s}\right\}\|v\|_{1, s} \\
& \leqq C h^{s}\|v\|_{1, s},
\end{aligned}
$$

which proves the theorem.

4. Smoothing of Initial Data. In this section, we adapt to the present context the analysis in [8]. We begin by introducing a modification of the definition of a smoothing operator in that paper: A family of linear translation invariant operators $M_{h}, 0<h \leqq h_{0}$, which is uniformly bounded in $L_{\infty}$, is called a family of smoothing 
operators of orders $(\mu, \nu)$ if, for some number $\delta$ with $0<\delta \leqq \pi / 2$, there are linear translation invariant operators $B_{h, \alpha}{ }^{(0)}(|\alpha|=\mu)$ and $B_{h, \alpha}{ }^{(1)}(|\alpha|=\nu)$ which are uniformly bounded in $L_{1}$ for $h \leqq h_{0}$ and such that

$$
\begin{array}{ll}
M_{h} v=v+h^{\mu} \sum_{|\alpha|=\mu} D^{\alpha} B_{h, \alpha}^{(0)} v & \text { if } \hat{v}(\xi)=0 \text { for } h|\xi|>2 \delta, \\
M_{h} v=h^{\prime} \sum_{|\alpha|=\nu} \partial_{h}^{\alpha} B_{h, \alpha}^{(1)} v & \text { if } \hat{v}(\xi)=0 \text { for } h|\xi|<\frac{1}{2} \delta .
\end{array}
$$

(Recall that $\partial_{h}{ }^{a}$ denotes divided forward differences.)

The difference compared to the definition in [8] consists in the fact that here we allow two parameters $\mu$ and $\nu$, whereas in [8] it was assumed that $\mu=\nu$. It is easy to show, for instance, that in one dimension the averaging operator

$$
\begin{aligned}
M_{h} v(x) & =h^{-1} \int_{-h / 2}^{h / 2} v(x-y) d y \\
& =\mathcal{F}^{-1}\left(\frac{2 \sin \frac{1}{2} h \xi}{h \xi}\right) \mathfrak{F v}
\end{aligned}
$$

is of orders $(2,1)$, and the weighted averaging operator

$$
M_{h} v(x)=h^{-1} \int_{-h}^{h}\left(1-\left|h^{-1} y\right|\right) v(x-y) d y=\mathcal{F}^{-1}\left(\frac{2 \sin \frac{1}{2} h \xi}{h \xi}\right)^{2} F_{v}
$$

has orders $(2,2)$.

The main result in [8] is then that under the general assumptions of Section 3, if $T$ is positive and if $M_{h}$ has orders $(\mu, \mu)$ where $\mu$ is the order of accuracy of the difference operator, then there exists a constant $C$ such that, for $0 \subseteq \mathfrak{e}_{0}$,

$$
\left\|E_{k}^{n} M_{h} v-E(n k) v\right\|_{\infty} \leqq C h^{\mu}(n k)^{-\mu /(2 M)}\|v\|_{\infty}, \quad 0 \leqq n k \leqq T .
$$

We shall now prove a result which shows that in certain cases the second smoothing parameter $\nu$ may be reduced without loss of convergence rates. In particular, if we consider the function $\chi_{\sigma}$ of Example 1.1 with $0<\sigma<1$ and a difference operator of accuracy $\mu=2$, then (4.3) shows that we get second-order convergence for times bounded away from zero by applying first the smoothing operator (4.2) of orders $(2,2)$, whereas the following theorem shows that second-order convergence is attained already by the operator $(4.1)$ of orders $(2,1)$.

THEOREM 4.1. Let the general assumptions of Section 3 hold. Let $d<s \leqq \mu$, $0<T_{0}<T_{1}$, and assume that $M_{h}$ has orders $(\mu, \nu)$, where $\mu$ is the order of accuracy of the difference operator and $\nu \geqq \mu-s$. Then there exists a constant $C$ such that, for $v \in B_{1}^{*}$,

$$
\left\|E_{k}^{n} M_{h} v-E(n k) v\right\|_{\infty} \leqq C h^{\mu}\|v\|_{1, s}, \quad T_{0} \leqq n k \leqq T_{1} .
$$

Proof. Consider first $j$ such that $h 2^{i} \leqq \delta$. Using Lemma 3.4 and the fact that $M_{h}$ is bounded and translation invariant, and the same argument as in (3.4), we get

$$
\begin{aligned}
\left\|\left(E_{k}^{n}-E(n k)\right) M_{h} v_{i}\right\|_{\infty} & \leqq C h^{\mu}\left\|M_{h} v_{i}\right\|_{\infty} \leqq C h^{\mu}\left\|v_{i}\right\|_{\infty} \\
& \leqq C h^{\mu} 2^{-i(s-d)}\|v\|_{1, s} .
\end{aligned}
$$

For these $j$, using (i) and Lemma 3.2 we further have (note that since $\operatorname{supp}\left(B_{h, \alpha}{ }^{(0)} v_{i}\right)$ is compact, $D^{\alpha} B_{h, \alpha}{ }^{(0)} v_{i}$ exists) 


$$
\begin{aligned}
\left\|E(n k)\left(M_{h}-I\right) v_{i}\right\|_{\infty} & \leqq C h^{\mu} \sum_{|\alpha|=\mu}\left\|E(n k) D^{\alpha} B_{h, \alpha}^{(0)} v_{i}\right\|_{\infty} \\
& \leqq C h^{\mu} \sum_{|\alpha|=\mu}\left\|B_{h, \alpha}^{(0)} v_{j}\right\|_{1} \leqq C h^{\mu}\left\|v_{i}\right\|_{1} \leqq C h^{\mu} 2^{-i \bullet}\|v\|_{1,2}
\end{aligned}
$$

Together, these estimates imply that, for $h 2^{i} \leqq \delta$,

$$
\left\|\left(E_{k}^{n} M_{h}-E(n k)\right) v_{i}\right\|_{\infty} \leqq C h^{\mu} 2^{-i(s-d)}\|v\|_{1, s} .
$$

To estimate the same quantity for $h 2^{i}>\delta$, we first use (ii) and Lemmas 3.3 and 3.1 , and get

$$
\begin{aligned}
\left\|E_{k}^{n} M_{h} v_{j}\right\|_{\infty} & \leqq C h^{\nu} \sum_{|\alpha|=\nu}\left\|E_{k}^{n} \partial_{h}^{\alpha} B_{h, \alpha}^{(1)} v_{j}\right\|_{\infty} \leqq C h^{\nu} \sum_{|\alpha|=\nu}\left\|B_{h, \alpha}^{(1)} v_{i}\right\|_{(h)} \\
& \leqq C h^{\nu} \sum_{|\alpha|=\nu ;|\beta| \leqq d} h^{|\beta|}\left\|D^{\beta} B_{h, \alpha}^{(1)} v_{i}\right\|_{1} \leqq C h^{\nu}\left(h 2^{i}\right)^{d}\left\|v_{i}\right\|_{1} \\
& \leqq C h^{\mu-s+d} 2^{-i(s-d)}\|v\|_{1, s} .
\end{aligned}
$$

Let $\Delta$ denote the Laplacian, and let $m$ be a nonnegative integer with $2 m+s \geqq \mu$. By Lemmas 3.2 and 1.1 we then also obtain, for $h 2^{i}>\delta$,

$$
\begin{aligned}
\left\|E(n k) v_{i}\right\|_{\infty} & \leqq\left\|E(n k) \Delta^{m} \mathfrak{F}^{-1}\left(|\xi|^{-2 m} \hat{v}_{i}\right)\right\|_{\infty} \\
& \leqq C\left\|\mathcal{F}^{-1}\left(|\xi|^{-2 m} \hat{v}_{j}\right)\right\|_{1} \leqq C \sum_{l=j-1}^{i+1} M\left(\varphi_{l}|\xi|^{-2 m}\right)\left\|v_{i}\right\|_{1} \\
& \leqq C 2^{-(2 m+s) ;}\|v\|_{1, s} \leqq C 2^{-\mu j}\|v\|_{1, s} .
\end{aligned}
$$

Altogether, we obtain, by (4.4), (4.5) and (4.6),

$$
\begin{aligned}
\left\|E_{k}^{n} M_{h} v-E(n k) v\right\|_{\infty} & \\
& \leqq C\left\{h^{\mu} \sum_{h 2^{i} \leqq \delta} 2^{-i(s-d)}+h^{\mu-s+d} \sum_{h 2^{i}>\delta} 2^{-i(s-d)}+\sum_{h 2^{i}>\delta} 2^{-\mu i}\right\}\|v\|_{1, s} \\
& \leqq C h^{\mu}\|v\|_{1, s},
\end{aligned}
$$

which concludes the proof of the theorem.

Department of Mathematics

Chalmers Institute of Technology and

The University of Göteborg

S-40220 Göteborg, Sweden

Department of Mathematics

University of Chicago

Chicago, Illinois 60637

1. N. K. Bari, Trigonometric Series. Vol. 2, Fizmatgiz, Moscow, 1961; English transl., Macmillan, New York; Pergamon Press, Oxford, 1964. MR 23 \#A3411; MR 30 \#1347.

2. A. Friedman, Partial Differential Equations of Parabolic Type, Prentice-Hall, Englewood Cliffs, N.J., 1964. MR 31 \#6062.

3. B. GREVHOLM, "On the structure of the spaces $\mathscr{L}_{k}{ }^{p, \lambda}$," Math. Scand., v. 26, 1970, pp. 241-254. MR 43 \#903.

4. L. Hörmander, Linear Partial Differential Operators, Die Grundlehren der math. Wissenschaften, Band 116, Academic Press, New York; Springer-Verlag, Berlin, 1963. MR 28 \#4221. 
5. F. JoHN, "On integration of parabolic equations by difference methods. I. Linear and quasi-linear equations for the infinite interval," Comm. Pure Appl. Math., v. 5, 1952, pp. 155-211. MR 13, 947.

6. M. L. Juncosa \& D. M. Young, "On the Crank-Nicolson procedure for solving parabolic partial differential equations," Proc. Cambridge Philos. Soc., v. 53, 1957, pp. 448461. MR 19, 583.

7. G. W. Hedstrom, "The rate of convergence of some difference schemes," J. SIAM Numer. Anal., v. 5, 1968, pp. 363-406. MR 37 \#6051.

8. H. O. KREISS, V. ThOMÉE \& O. WIDlund, "Smoothing of initial data and rates of convergence for parabolic difference equations," Comm. Pure Appl. Math., v. 23, 1970, pp. 241-259. MR 40 \#5160.

9. J. Löfström, "Besov spaces in the theory of approximation," Ann. Mat. Pura Appl., (4), v. 85, 1970, pp. 93-184. MR 42 \#2234.

10. J. PeEtre \& V. ThOMÉE, "On the rate of convergence for discrete initial-value problems," Math. Scand., v. 21, 1967, pp. 159-176. MR 40 \#8292.

11. W. WASOW, "On the accuracy of implicit difference approximations to the equation of heat flow," Math. Tables Aids Comput., v. 12, 1958, pp. 43-55. MR 20 \#6795.

12. O. Widlund, On the Rate of Convergence for Parabolic Difference Schemes. I. Numerical Solution of Field Problems in Continuum Physics, SIAM-AMS Proc., vol. II, Amer. Math. Soc., Providence, R.I., 1970, pp. 60-73. MR 41 \#9458a.

13. O. WIDLUND, "On the rate of convergence for parabolic difference schemes. II," Comm. Pure Appl. Math., v. 23, 1970, pp. 79-96. MR 41 \#9458b. 